Jacques TASSIN ${ }^{1}$

Ronald BELLEFONTAINE ${ }^{2}$

Edmond ROGER ${ }^{3}$

Christian KuLl ${ }^{4}$

\section{Evaluation préliminaire des risques d'invasion par les essences forestières introduites à Madagascar}

${ }^{1}$ Cirad, UR 37, TA 10/D

34398 Montpellier cedex 5

France

${ }^{2}$ Cirad, UR 39, TA 10/D

34398 Montpellier cedex 5

France

3 Faculté des sciences

Université d'Antananarivo

Madagascar

${ }^{4}$ School of geography and environmental science Monash University Melbourne Australie

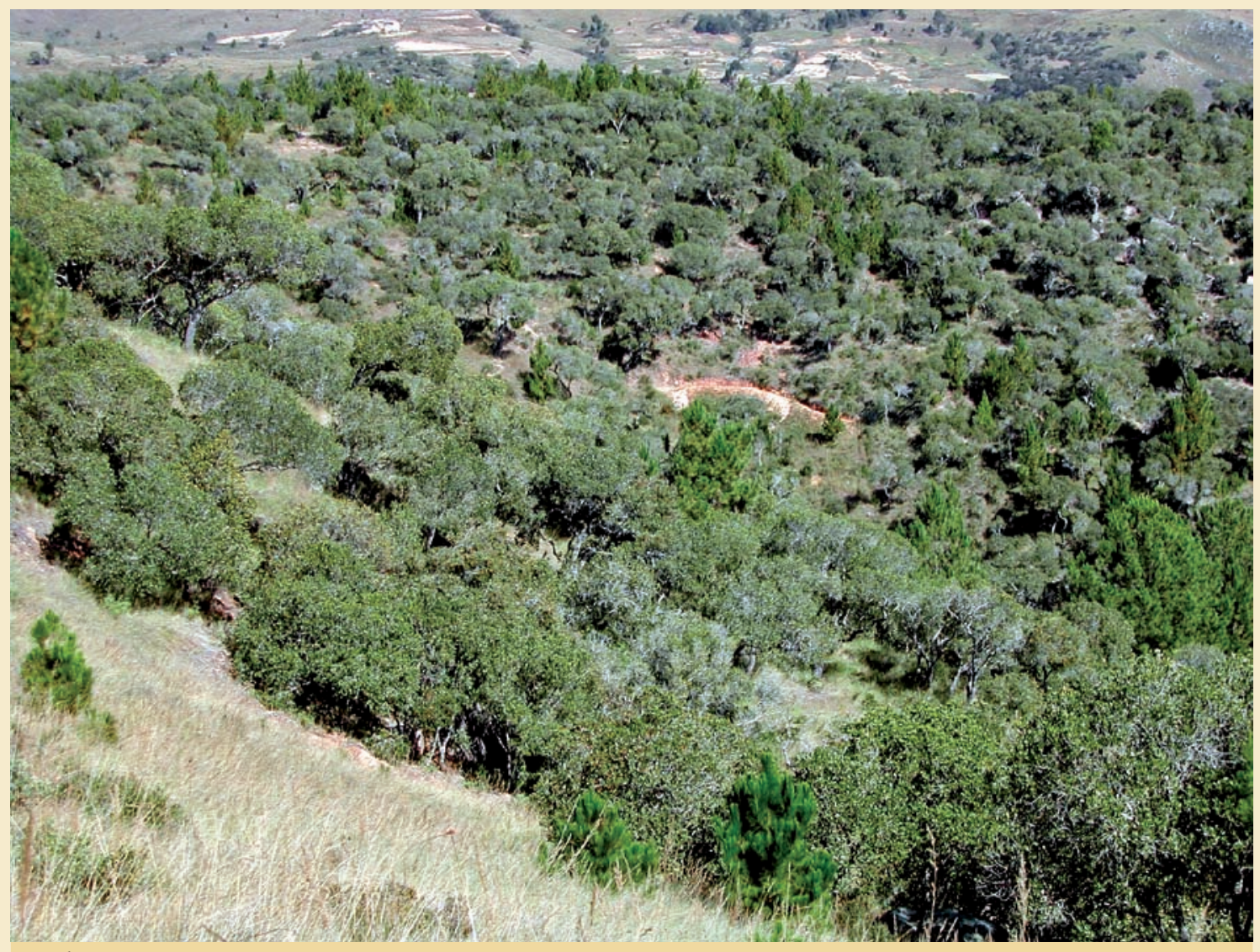

Photo 1.

Forêt dégradée à Uapaca bojeri, envahie par des pins dans la commune d'Ambohimanjaka-Sahatsiho, à $200 \mathrm{~km}$ au sud d'Antananarivo. Photo C. Kull. 
J. TASSIN, R. BELLEFONTAINE,

E. ROgER, C. KULL

\section{RÉSUMÉ}

\section{ÉVALUATION PRÉLIMINAIRE DES RISQUES D'INVASION PAR LES ESSENCES FORESTIÈRES INTRODUITES à MADAGASCAR}

Il est désormais admis que l'introduction d'une espèce exotique en milieu insulaire s'accompagne toujours d'un risque environnemental, représenté par l'éventuel déclenchement d'une invasion biologique. À Madagascar, ce risque est d'autant plus élevé que les introductions de matériel végétal s'accroissent à un degré très supérieur à celui des actions de lutte préventive, que la fréquence et l'intensité des perturbations humaines facilitent les invasions, et que la biodiversité à protéger est remarquable. Un examen préliminaire des 406 introductions d'arbres et arbustes effectuées sur cette île à des fins forestières ou agroforestières révèle que $12,8 \%$ de ces espèces se sont avérées nuisibles dans d'autres pays, et que 39,2 \% présentent un risque potentiel pour Madagascar dans la mesure où elles sont au moins susceptibles de s'y naturaliser. Une liste de 19 espèces présentant un risque élevé d'invasion est présentée. Sept d'entre elles sont connues pour avoir, à la fois, une reproduction sexuée et une multiplication végétative par drageonnage importantes. Face à une telle menace, un plan de gestion s'impose sur le plan national de manière à hiérarchiser, puis mettre en œuvre les actions de communication, de recherche, de lutte préventive et de détection précoce qui s’imposent.

Mots-clés : biodiversité, drageonnage, espèce exotique, espèce invasive, insularité, Madagascar.

\section{ABSTRACT}

\section{A PRELIMINARY ANALYSIS OF THE INVASIVENESS OF INTRODUCED FOREST SPECIES IN MADAGASCAR}

It is now well-known that introducing exotic species to an island carries environmental risks, as this can trigger a biological invasion. In Madagascar, the risk is aggravated by three factors: plant material is being introduced much more rapidly than any programmes to prevent invasion; the degree and frequency of human disturbance is facilitating plant invasions; biodiversity on the island is under severe threat. Our preliminary analysis of the status of 406 introduced species in Madagascar found that $12.8 \%$ were invasive in other countries, and that $39.2 \%$ carried an environmental risk of potential naturalisation. This article describes a shortlist of 19 species with a high environmental risk factor. Seven of these are known to reproduce both sexually and vegetatively, by suckering. To deal with the threat of invasion, a national management plan is needed to set priorities and then implement programmes for essential communication, research, prevention and early detection.

Keywords: biodiversity, suckering, exotic species, invasive species, insularity, Madagascar.

\section{RESUMEN}

\section{EVALUACIÓN PRELIMINAR DE RIESGOS POR LA INVASIÓN DE ESPECIES FORESTALES INTRODUCIDAS EN MADAGASCA}

Hoy sabemos que la introducción de una especie exótica en un medio insular siempre viene acompañada de un riesgo ambiental, representado por el posible desencadenamiento de una invasión biológica. En Madagascar, este riesgo mucho mayor porque las introducciones de material vegetal se incrementan a un ritmo mucho más alto que el de las acciones de control preventivo, porque la frecuencia e intensidad de las perturbaciones antrópicas facilitan la invasión, y porque la biodiversidad que hay que proteger es excepcional. Un examen preliminar de las 406 introducciones de árboles y arbustos efectuadas en esta isla con fines forestales o agroforestales refleja que el $12,8 \%$ de dichas especies se revelaron perjudiciales en otros países, y que el 39,2\% presenta un riesgo potencial ya que podrían naturalizarse. Se expone una lista de 19 especies que presentan un alto riesgo de invasión. Siete de ellas son conocidas por tener, a la vez, unas importantes capacidades de reproducción sexual y propagación vegetativa por retoñado. Ante tal amenaza, hay que disponer de un plan nacional de manejo para jerarquizar y, posteriormente, implementar las necesarias acciones de comunicación, investigación, control preventivo y detección precoz.

Palabras clave: biodiversidad, retoñado, especie exótica, especie invasiva, insularidad, Madagascar. 


\section{Introduction}

Les invasions biologiques constituent une cause majeure de l'affaiblissement de populations d'espèces indigènes, tout particulièrement en milieu insulaire. Le contrôle des espèces dites invasives représente à ce titre une composante première du développement durable, dans un contexte de redistribution croissante du vivant consécutive à la mondialisation des échanges (VITOUSEK et al., 1997). On entend ici par invasive toute espèce qui, à la suite de son introduction, prolifère, s'étend et persiste au détriment des écosystèmes et des espèces indigènes (MAck et al., 2000). Parmi les plantes invasives, les essences ligneuses sont les plus dommageables au fonctionnement des écosystèmes (BINGGELI, 1996 ; CRONK, FulLER, 1997 ; REJMANEK et al., 2005) (figure 1). De ce fait, les risques d'invasions végétales inhérents à l'introduction d'espèces ligneuses utilisées dans des programmes forestiers ou agroforestiers ont fait l'objet d'une récente prise de conscience internationale (BINGGELI, HAMILTON, 1993 ; RICHARDSON, 1998). Certains genres botaniques utilisés en foresterie sont aujourd'hui reconnus comme présentant des risques d'invasion majeurs, tels que Acacia, Acer, Albizia, Cedrela, Eucalyptus, Grevillea, Leucaena, Maesopsis, Melaleuca, Pinus, Prosopis, Robinia, Sesbania, Swietenia et Toona (TASSIN et al., 2007).

Une simple mise en quarantaine ne peut suffire pour évaluer de tels risques, dans la mesure où, chez les espèces ligneuses, plusieurs dizaines d'années peuvent séparer l'introduction de l'invasion (KowArIK, 1995 ; CRONK, FulLer, 1997 ; ReICHARd, HAMIlton, 1997). Aussi, à des fins préventives, les introductions d'espèces ligneuses ont-elles donné lieu à la mise au point de systèmes d'évaluation ex ante du risque d'invasion représenté, dont l'efficacité n'a cessé de s'accroître ces dernières années (REICHARD, HAMILTON, 1997 ; DAEHLER et al., 2004 ; REJMANEK et al., 2005 ; THUILLER et al., 2005).

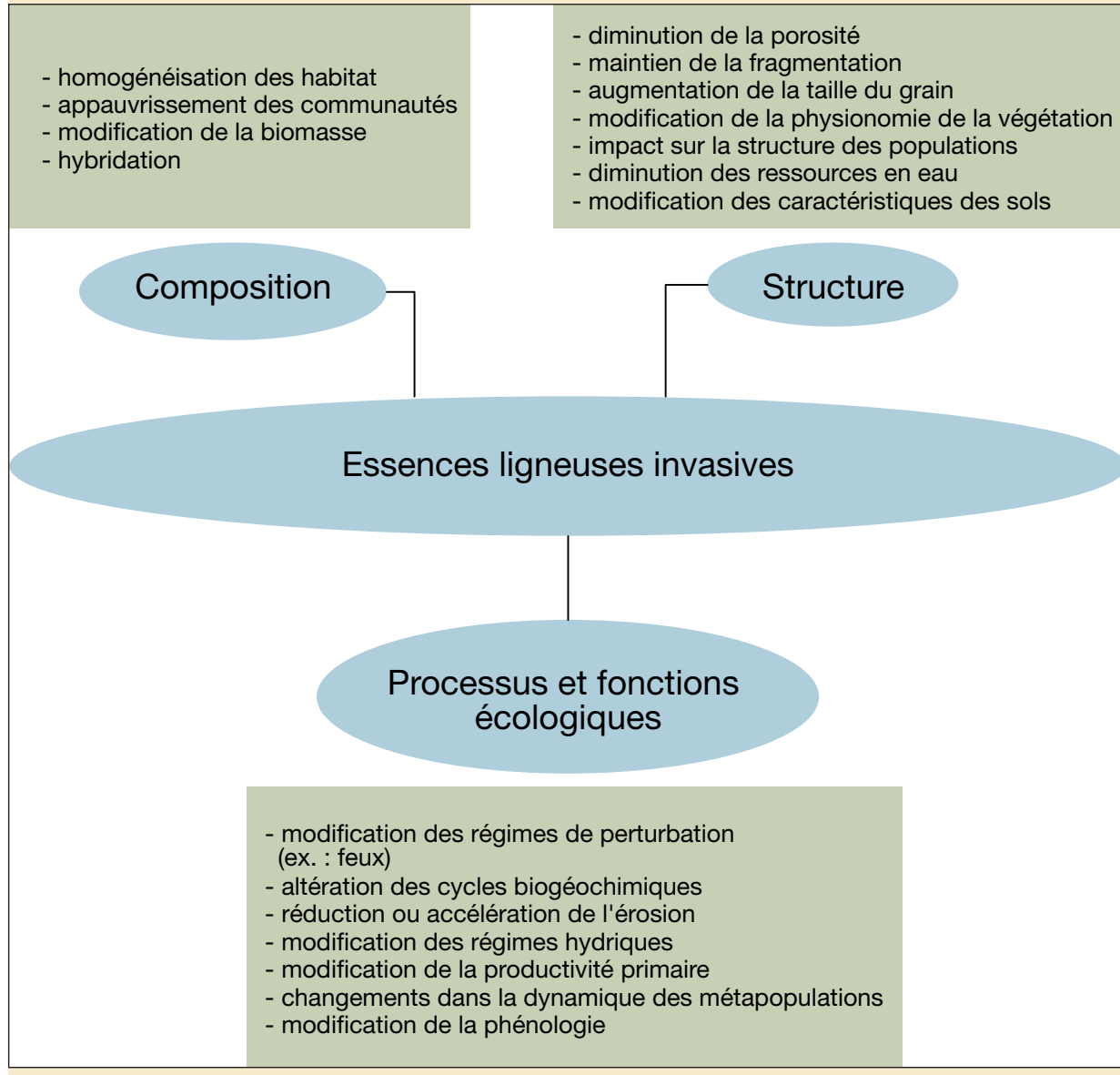

Figure 1.

Modes d'impact des essences ligneuses invasives sur les écosystèmes terrestres (d'après Noss, 1990 ; CRONK, Fuller, 1997 ; WALKER, SMITH, 1997).

Au sein de ces systèmes de prédiction, le fait que l'espèce évaluée se soit déjà révélée invasive en un lieu représente une part très significative du caractère prédictif de tels outils (DAEHLER, 2004 ; REJMANEK et al., 2005 ; REICHARD, HAMILTON, 1997 ; TASSIN et al., 2007). De manière complémentaire, la pression d'introduction apparaît également comme un facteur de prédiction essentiel. Dans le domaine animal, ce facteur se situe au premier rang des critères de prédiction des invasions de vertébrés (JeSCHKE, StRAYER, 2006). La pression d'introduction traduit l'intensité avec laquelle l'introduction d'une espèce a été entreprise et peut donc s'exprimer sous différentes formes. Dans le règne végétal, elle peut être caractérisée par le nombre de plantations réalisées ou la surface totale de ces plantations (KrIVANEK, PYSEK, 2006).
À Madagascar, les risques environnementaux majeurs que représentent les feux et les défrichements ont contribué à occulter les risques liés aux invasions de plantes, bien qu'ils aient été envisagés depuis presqu'un siècle (PERRIER DE LA BÂTHIE, 1928). Tel l'arbre cachant la forêt, l'invasion extrêmement spectaculaire impliquant plusieurs espèces d'opuntias (photo 2) dans les régions arides de l'île a probablement contribué à masquer les autres invasions végétales. À notre connaissance, en dehors d'une revue récente sur la question (BINGGELI, 2004), aucune étude n'a porté spécifiquement sur les conséquences environnementales des invasions de plantes à Madagascar. À l'inverse, l'histoire des introductions est assez bien documentée, notamment celle des 


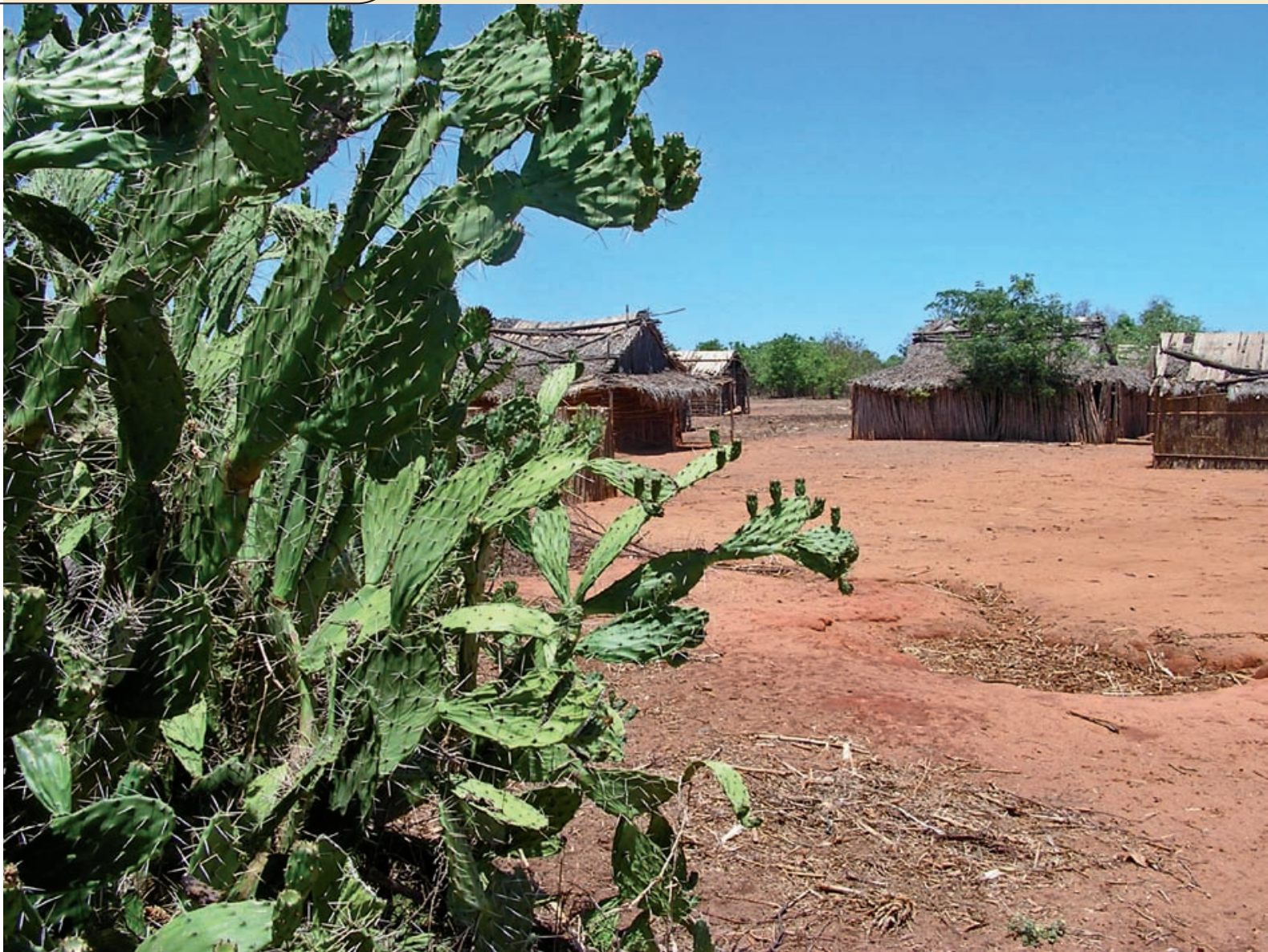

Photo 2.

L'invasion végétale la plus spectaculaire à Madagascar est représentée par plusieurs espèces d'opuntias introduites dès le XVIII siècle par les Européens. Photo J. Tassin.

espèces forestières et agroforestières (GACHET, 1966 ; ChAuVET, 1968 ; Sutter, Rakotonoely, 1989). À défaut de disposer d'informations sur les surfaces totales plantées, le nombre d'arboretums abritant chaque essence forestière peut, dès lors, être considéré comme un indicateur permettant d'approcher cette pression d'introduction.

L'objet de cet article est d'obtenir une hiérarchisation préliminaire simple des risques d'invasion représentés par des essences forestières introduites à Madagascar, en se référant à deux critères principaux : le nombre de pays où l'espèce en question s'est révélée invasive, et le nombre d'arboretums où elle a été introduite. En l'absence d'informations plus complètes, il n'est malheureusement pas possible d'appliquer les systèmes d'évaluation ex ante cidessus évoqués.

\section{Matériel ef méthodes}

\section{Les introductions d'espèces forestières}

Les premières introductions répertoriées d'essences forestières à Madagascar datent des années 18901895 et concernent essentiellement Antananarivo, Antsirabe et Fianarantsoa ; elles restent malheureusement très mal documentées (CHAUVET, 1968). Les premières datations précises portent sur les introductions réalisées dans la station d'Analamazaotra, ou Périnet (préfecture de Moramanga), avec certains pins (ex. : Pinus kesyia) et eucalyptus (ex. : Eucalyptus robusta) introduits entre 1914 et 1920 (Chauvet, 1968). Des références de plantations d'eucalyptus plus anciennes existent (ex. : planta- tions le long de la route AntananarivoToamasina entre 1897 et 1900), mais le nom de ces espèces n'est, hélas, presque jamais précisé dans la documentation ancienne. Plus tardivement, à partir de 1928, des essais d'introduction de pins ont été réalisés à Manjakatompo, sur les pentes du massif de l'Ankaratra (GACHET, 1966).

Mais c'est essentiellement entre 1950 et 1956 que, à la faveur d'un renforcement du Service des Eaux et Forêts et de la création concomitante de nombreux arboretums (figure 2), la vague d'introduction d'essences forestières exotiques a connu son point culminant à Madagascar (GACHET, 1962, 1965 et 1966 ; CHAUVET, 1968 ; SUtTer, RAKotonoely, 1989). Dans le seul arboretum d'Ampamaherana (préfecture de Fianarantsoa), près de 150 espèces ont été introduites (CHAUVET, 1968). On observe ici que, du fait de sa très 


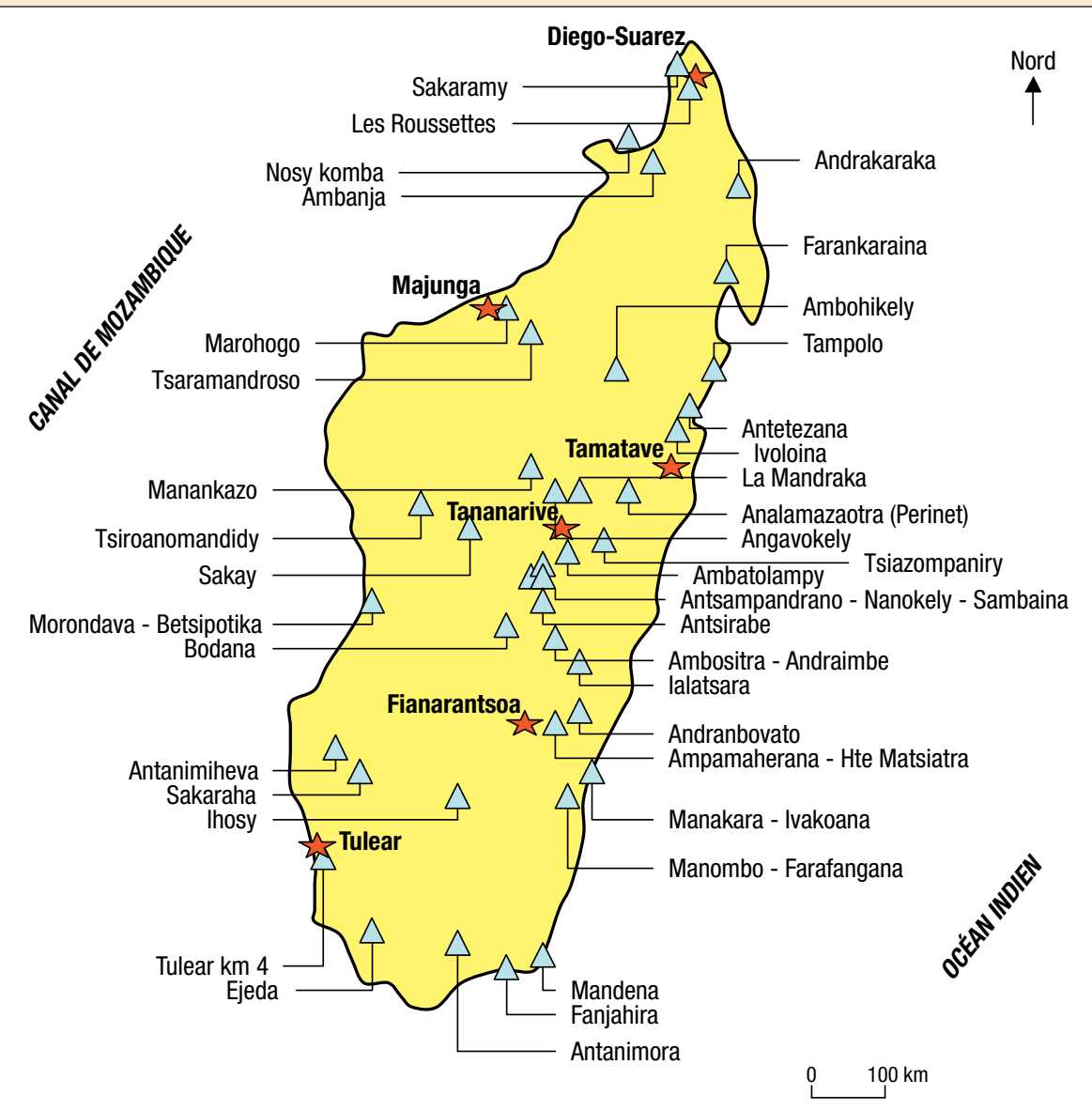

Figure 2.

Carte des arboretums de Madagascar.

faible variabilité, la date d'introduction ne peut dès lors être utilisée comme un critère de prédiction des invasions d'espèces forestières à Madagascar, comme cela a pu être analysé en d'autres pays (KRIVANEK et al., 2006).

\section{Recueil de données}

Pour inventorier les introductions d'essences forestières réalisées à Madagascar, nous avons principalement exploité des documents de synthèse dressant le bilan des premières plantations expérimentales réalisées au sein d'arboretums (GACHET, 1966 ; Chauvet, 1968 ; Sutter, RakotoNOELY, 1989). Ces données sont nécessairement incomplètes, dans la mesure où de telles analyses ne font pas toujours mention des introductions ayant échoué. En outre, certains arboretums, tel celui de Sakaramy, dans la préfecture de Diego-Suarez, ou celui de Marohogo (préfecture de Majunga), ont été en partie détruits par le feu (Chauvet, 1968). Néanmoins, un grand nombre de ces introductions restent documentées de manière satisfaisante.

En particulier, CHAUVET (1968) a établi un bilan exhaustif des introductions d'espèces réalisées dans 31 arboretums ou stations forestières de Madagascar (figure 2). Les espèces synonymes ou pour lesquelles nous n'avons pu déterminer l'appellation actuelle avec certitude ont été soustraites, à l'instar de ChAuvet (1968) évoquant d'ailleurs lui-même certaines confusions possibles dans son ouvrage. Nous avons ainsi établi une liste de 406 espèces correctement nommées, introduites dans leur très grande majorité avant même la fin des années 1950 (tableau I).

\section{Méthodologie préliminaire d'analyse des risques}

Dans le cadre de cette approche préliminaire des risques d'invasion, nous avons alors recouru à une méthode très simple de hiérarchisation des espèces, en faisant valoir les éléments dont nous disposions. Parmi ces 406 espèces introduites, nous avons tout d'abord identifié celles qui étaient réputées invasives dans d'autres pays, en nous référant principalement à deux bases de données : The Global Compendium of Weeds ${ }^{1}$, et Invasive Woody Plants - World Species List $^{2}$. Parallèlement, nous avons dénombré les arboretums dans lesquels chacune de ces espèces avait été plantée, en nous référant à l'ouvrage de CHAUVET (1968). Le nombre d'arboretums représentés par chaque espèce constitue un indicateur supposé satisfaisant de la pression d'introduction qui s'y rattache.

Les espèces ont été triées de manière décroissante par nombre de pays envahis, puis par nombre d'arboretums où elles ont été plantées. Ce tri, certes imparfait car fondé sur deux critères, aussi prépondérants soient-ils, permet d'identifier un groupe d'espèces rassemblant des essences à haut risque d'invasion.

\section{Résultats}

Parmi les 406 espèces introduites figurent 62 mimosacées (dont 40 acacias), 26 papilionacées et 22 césalpiniacées, soit un ensemble de 110 légumineuses $(27,1 \%)$. La part des myrtacées et des pinacées est également très importante, avec 115 myrtacées, dont 110 eucalyptus, et 40 pinacées recouvrant 36 pins. Eucalyptus et pins représentent $36,0 \%$ des espèces introduites. La consultation des bases de données sur les espèces invasives montre que, en d'autres pays, 52 de ces espèces $(12,8 \%)$ sont considérées comme nuisibles pour l'environnement et 107 autres espèces $(26,4 \%)$ sont notées naturalisées. Au total, au sein 


\section{Discussion}

Tableau I.

Liste de 21 espèces forestières qui présentent un risque élevé d'invasion à Madagascar (cf. Matériel et méthodes pour le dénombrement des pays et arboretums). Les espèces qui ont une aptitude au drageonnage sont signalées par $a$.

Espèce

Pinus pinaster Aiton

Robinia pseudoacacia L. a

Acacia melanoxylon R. Br. a

Melia azedarach L. a

Pinus patula Schiede ex-Schltdl. \& Cham.

Cinnamomum camphora (L.) J. Presl a

Pinus radiata $\mathrm{D}$. Don

Acacia longifolia (Andrews) Willd.

Pinus caribaea Morelet

Pinus taeda L.

Grevillea banksii R. Br.

Jacaranda mimosifolia D. Don

Maesopsis eminii Engl. a

Pinus halepensis Mill.

Pinus elliottii Engelm.

Acacia cyclops A. Cunn. ex-G. Don

Gleditsia triacanthos L. a

Pithecellobium dulce (Roxb.) Benth. a

Casuarina glauca Sieber ex-Spreng. a

Ilex aquifolium L.

Prosopis juliflora (Sw.) DC. a

du panel d'espèces investigué, 159 $(39,2 \%)$ présentent un risque potentiel pour Madagascar dans la mesure où elles sont au moins susceptibles de s'y naturaliser.

Chauvet (1968) signalait au demeurant que certaines de ces espèces étaient effectivement devenues invasives (ex. : Acacia dealbata, Albizia lebbeck, Albizia chinensis, Casuarina equisetifolia, Grevillea banksii, Leucaena leucocephala, Melaleuca leucodendron). SUTTER et RAKOTONOELY (1989) évoquaient de surcroît le caractère invasif de Jacaranda mimosifolia (photo 3) dans l'arboretum du lac Alaotra. En revanche, certaines espèces réputées invasives en d'autres régions du monde ont été mentionnées par Chauvet (1968) comme produisant peu de semences (Acacia saligna, Pinus patula). De même, certaines espèces, connues pour être particulièrement invasives en d'autres contrées,

\begin{tabular}{|c|c|}
\hline Pays & Arboretums \\
\hline 16 & 7 \\
\hline 11 & 1 \\
\hline 6 & 9 \\
\hline 5 & 5 \\
\hline 4 & 13 \\
\hline 4 & 10 \\
\hline 4 & 10 \\
\hline 4 & 4 \\
\hline 3 & 13 \\
\hline 2 & 12 \\
\hline 2 & 5 \\
\hline 2 & 5 \\
\hline 2 & 5 \\
\hline 2 & 4 \\
\hline 2 & 3 \\
\hline 2 & 2 \\
\hline 2 & 2 \\
\hline 2 & 2 \\
\hline 2 & 1 \\
\hline 2 & 1 \\
\hline 2 & 1 \\
\hline
\end{tabular}

n'ont été introduites que dans un petit nombre d'arboretums ou stations. C'est le cas de Robinia pseudoacacia, introduit uniquement sur la station de Manjakatompo (préfecture d'Ambatolampy) et ne donnant lieu qu'au développement d'arbres chétifs (CHAUVET, 1968).

De manière relativement arbitraire, et considérant qu'une invasion dans un seul pays pouvait relever d'une singularité non forcément reproductible à Madagascar, nous avons retenu les espèces réputées invasives dans au moins deux autres pays. Parmi les 21 espèces ainsi mises en avant, neuf sont invasives dans au moins trois autres pays, et douze le sont dans deux autres pays. On obtient une représentation visuelle du positionnement respectif des neuf espèces principales en les positionnant sur une figure dont les axes correspondent aux deux critères d'évaluation retenus (figure 3 , encadré 1 ).

\section{Confrontation aux données de terrain}

Parmi les 21 espèces listées dans le tableau I, les observations de terrain montrent que cinq $(23,8 \%)$ d'entre elles se révèlent aujourd'hui particulièrement invasives : Cinnamomum camphora, Grevillea banksii, Melia azedarach, Pinus patula et Pithecellobium dulce (R. Edmond, obs. pers.).

Les plaquettes publicitaires présentant l'huile de Raventsara, distillée à partir des feuilles de Cinnamomum camphorum, désignent communément cette espèce comme poussant désormais à l'état sauvage. Grevillea banksii forme des peuplements monospécifiques très étendus à l'ouest d'AmbilaLemaitso (BıngGELI, 2004) et colonise aujourd'hui abondamment certains lavaka du lac Alaotra où il a été utilisé dans les années 1950 et 1960 dans le cadre de programmes de défense et de restauration des sols (TASSIN, 1995). Melia azedarach est communément naturalisé dans les Hautes Terres, où il apparaît souvent sous la forme de petits bosquets. Pithecellobium dulce (photo 4), probablement introduit pour la constitution de haies vives et la production de fourrage, est devenu invasif au sud-est de l'île, notamment dans la réserve de Berenty, où on l'observe le long de forêts-galeries (BINGGELI, 2004). Il est également abondamment naturalisé dans la région de Morondava, où on le rencontre préférentiellement au bord de terrains humides (J. TASSIN, obs. pers.).

La capacité de colonisation de terrains perturbés par Pinus patula a été, quant à elle, rapidement observée par les forestiers qui notèrent « la présence de semis naturels le long des chemins, sur les talus, sur les éboulis ", au sein des périmètres de reboisement de la Haute-Matsiatra (VIGnAL, 1956). Toutefois, la dénomination des pins à Madagascar reste en partie incertaine, et il est probable que, au-delà de l'espèce $P$. patula, ce soit en fait une bonne partie des espèces de pins qui sont devenues invasives à la faveur des feux de brousse qui, lorsqu'ils sont fugaces, favorisent la germination des graines. 


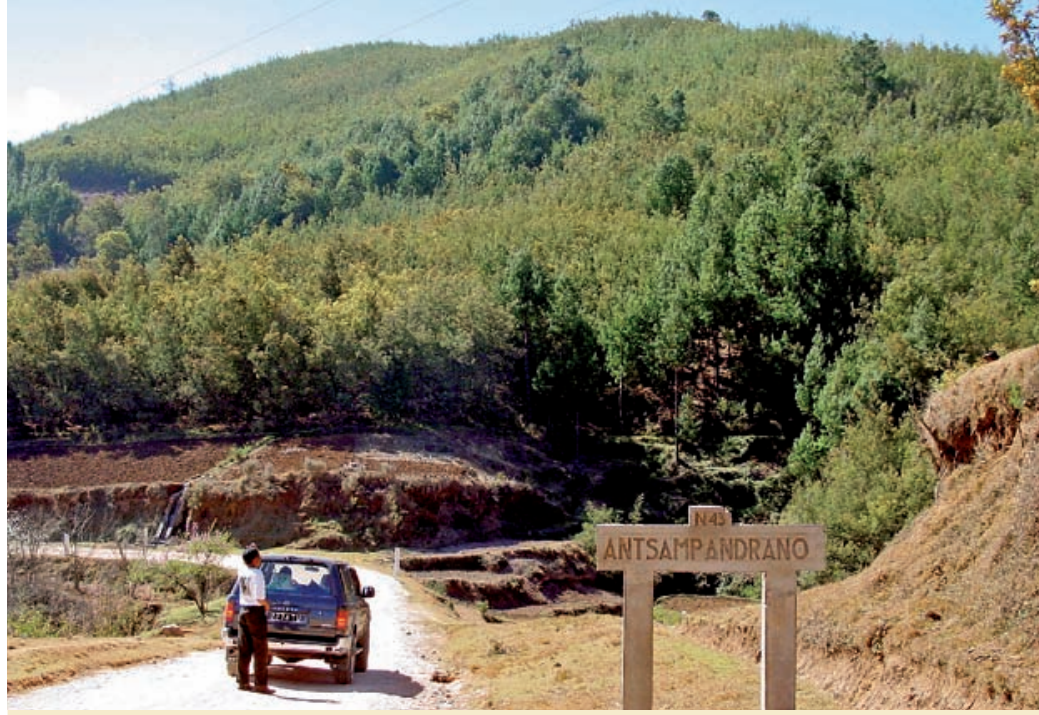

Photo 6.

Acacia dealbata, très invasif sur les Hauts-Plateaux (vue d'une colline envahie à Antsampandrano), n'en constitue pas moins une ressource en bois de feu. Photo C. Kull.

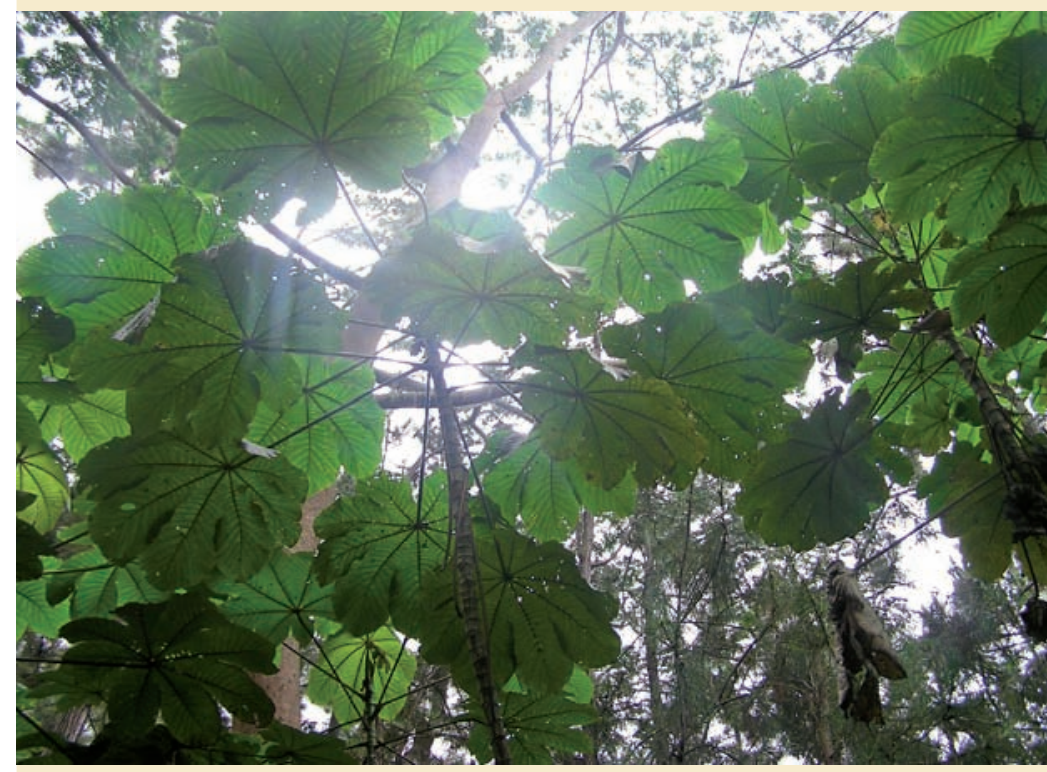

Photo 7.

Cecropia peltata abonde aujourd'hui dans la partie orientale, sous couverts forestiers dégradés. Photo J. Tassin.

La pression d'introduction des espèces forestières communément plantées demande, à ce titre, à être affinée. D'autres critères, fondés sur les traits de vie et l'autécologie des espèces, pourront également être intégrés à des modèles de prédiction de la sorte rendus plus performants.

Une seconde étape pourrait conduire à inventorier les régions en cours d'invasion souvent dégradées par les activités humaines, et notamment celles qui sont les plus atteintes. Dès lors, on pourrait établir une carte de distribution potentielle par espèce invasive en fonction des sites préférentiellement colonisés (pédologie, topographie, exposition, bilan hydrique du sol). Il serait, alors, avantageux de former des patrouilles d'agents de terrain pour réduire au stade précoce l'avancée de certaines espèces invasives. Les espèces listées sur le tableau I mériteraient, à ce titre, une attention toute particulière.

Les invasions de plantes restent encore très mal renseignées à Madagascar (BINGGELI, 2004). Réunir les informations manquantes constitue un préalable indispensable pour dépasser ce type d'approche préliminaire dans l'évaluation des risques liés aux introductions d'essences ligneuses, et gagner ainsi en capacité de prédiction.

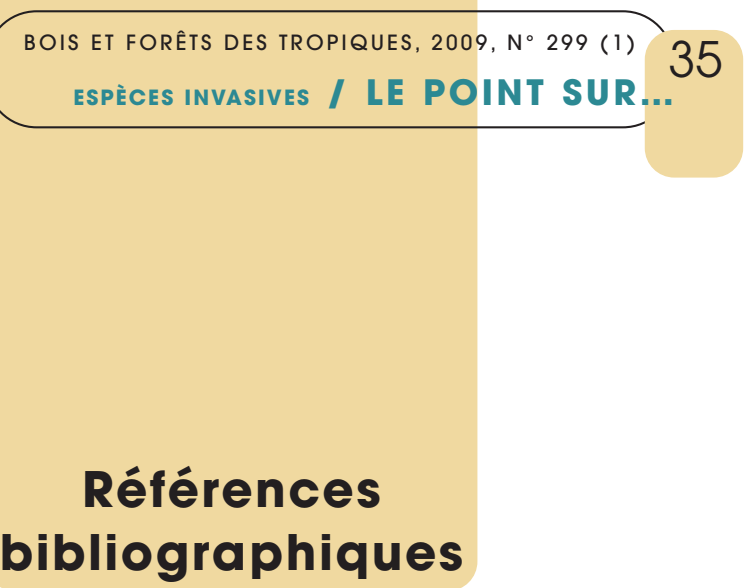

BELEM B., BOUSSIM J.I., BELLEFONTAINE R., GUINKO S., 2008. Stimulation du drageonnage de Bombax costatum Pelegr. et Vuillet par blessures de racines au Burkina Faso. Bois et Forêts des Tropiques, 295 : 71-79.

BELLEFONTAINE R., 2005 a. Pour de nombreux ligneux, la reproduction sexuée n'est pas la seule voie : analyse de 875 cas - Texte introductif, tableau et bibliographie. Sécheresse, revue électronique, $n^{\circ} 3^{\mathrm{E}}$, déc. 2005. Cf. www. secheresse.info/article. php3?id_article $=2344$

BELLEFONTAINE R., 2005 b. Régénération naturelle à faible coût dans le cadre de l'aménagement forestier en zones tropicales sèches en Afrique. Université du Québec, Montréal, Vertig0, 6 (2) : 1-15. Cf. www.vertigo. uqam.ca/vol6no2/framerevue.html

BINGGELI P., HAMILTON A.C., 1993. Biological invasion by Maesopsis eminii in the East Usambara forests, Tanzania. Opera Botanica Belgica, 121 : 229-235.

BINGGELI P., 1996. A taxonomic, biogeographical and ecological overview of invasive woody plants. Journal of Vegetation Science, 7 : 121-124.

BINGGELI P., 2004. Introduced and invasive plants. In : Goodman S.M. \& J.P. Benstead (éd.) The natural history of Madagascar. University Chicago Press, États-Unis, 257-268.

CHAUVET B., 1968. Inventaire des espèces forestières introduites à Madagascar. École Nationale Supérieure Agronomique, Antananarivo, Madagascar, $187 \mathrm{p}$.

CRONK C.B., FULLER J.L., 1995. Plant invaders : the threat to natural ecosystems. Chapman \& Hall, Royaume-Uni, 241 p. 
DAEHLER C.C., DENSLOW J.S., ANSARI S., KUO H-C., 2004. A risk assessment system for screening out invasive pest plants from Hawaii and other Pacific Islands. Conservation Biology, 18 : 360-368.

DAVIS M.A., THOMPSON K., 2002. "Newcomers" invade the field of invasion ecology : question the field's future. Bulletin of the Ecological Society of America, 84 : 196-197.

DOURMA M., GUELLY K.A., KOKOU K., BATAWILA K., WALLA K., BELLEFONTAINE R., AKPAGANA K., 2006. Multiplication par drageonnage d'Isoberlinia doka et $I$. tomentosa au sein des formations arborées du Nord-Togo. Bois et Forêts des Tropiques, $289:$ 49-57.

GACHET C., 1962. Note sur les essais sylvicoles de la region de Morondava - Betsipotika, Dabara, Ambalavao. Centre Technique Forestier Tropical, Madagascar, note technique.

GACHET C., 1965. L'arboretum d'Ambohikely - Lac Alaotra. Centre Technique Forestier Tropical, Madagascar, note 29.

GACHET C., 1966. Les essences de reboisement à Madagascar. Premiers résultats des essais effectués en arboretums et en reboisements extensifs. Centre Technique Forestier Topical, Madagascar, note 84.

JACQ F., HLADIK A., BELLEFONTAINE R., 2005. Dynamique d'un arbre introduit à Mayotte, Litsea glutinosa (lauraceae) : une espèce envahissante ? Revue d'Écologie (Terre et Vie), 60 : 21-32.

JESCHKE J.M., STRAYER D.L., 2006. Determinants of vertebrate invasion success in Europe and North America. Global Change Biology, 12 : 1608-1619.

KOWARIK I., 1995. Time lags in biological invasions with regard to the success and failure of alien species. In : Pysek P., Prach K., Rejmánek M., Wade P. M. (éd.). Plant invasionsgeneral aspects and special problems. SPB Academic Publishing, Pays-Bas, 15-38.
KRIVANEK M., PYSEK P., 2006. Predicting invasions by woody species in a temperate zone : a test of three risk assessment schemes in the Czech Republic (Central Europe). Diversity and Distributions, 12 : 319-327.

KRIVANEK M., PYSEK P., JAROSIK V., 2006. Planting history and propagule pressure as predictors of invasion by woody species in a temperate region. Conservation Biology, 20 : 1487-1498.

KULL C.A., TASSIN J., HARIPRIYA R., 2007. Multifunctional, scrubby, and invasive forests ? Wattles in the highlands of Madagascar. Mountain Research and Development, 27 (3) : 224-231.

MACK R.N., SIMBERLOFF D., LONSDALE W.M., EVANS H., CLOUT M., BAZZAZ F.A., 2000. Biotic invasions: causes, epidemiology, global consequences, and control. Ecological Applications, $10: 689-710$.

MEUNIER Q., BELLEFONTAINE R., BOFFA J.M., 2006. Le drageonnage pour la régénération d'espèces médicinales en Afrique tropicale : cas du Spathodea campanulata en Ouganda. Université du Québec, Montréal, Vertig0, 7 (2) : 1-6. Cf. www.vertigo.uqam.ca/

NEERGAARD A. DE, SAARNAK C., HILL T., KHANYILE M., BERZOSA A.M., BIRCH-THOMSEN T., 2005. Australian wattle species in the Drakensberg region of South Africa - an invasive alien or a natural resource ? Agricultural Systems, 85 (3) : 216-233.

NOSS R.F., 1990. Indicators for monitoring biodiversity : a hierarchical approach. Conservation Biology, 44 : 355-364.

PERRIER DE LA BÂTHIE H., 1928. Les pestes végétales à Madagascar. Bulletin Economique de Madagascar, 1 : 104-109.

REICHARD S.H., HAMILTON W., 1997. Predicting invasions of woody plants introduced into North America. Conservation Biology, 11 : 193-203.
REJMANEK M., RICHARDSON D.M., HIGGINS S.I., PITCAIRN M., GROTKOPP E., 2005. Ecology of invasive plants: state of the art. In: Invasive alien species: a new synthesis. Mooney H.A., Mc Neely J.A., Neville L., Schei P.J., Waage J. (éd.), Island Press, Washington, États-Unis, 368 p.

RICHARDSON D.M., 1998. Forestry trees as invasive aliens. Conservation Biology, 12 : 18-26.

SUTTER E., RAKOTONOELY J., 1989. Projet Inventaire des ressources ligneuses. Cirad, Centre Technique Forestier Tropical, Antananarivo, Madagascar, rapport d'activités, 23 p.

TASSIN J., 1995. La protection des bassins versants à Madagascar. Bois et Forêts des Tropiques, 246 (4) : 7-22.

TASSIN J., SARRAILH J.-M., RIVIÈRE JN., 2007. Essences forestières et invasions : des systèmes de prédiction de plus en plus fiables. Bois et forêts des tropiques, 292 : 71-79.

THUILLER W., RICHARDSON D.M., PYSEK P., MIDGLEY G.F., HUGHES G.O., ROUGET M., 2005. Niche-based modelling as a tool for predicting the risk of alien plant invasions at a global scale. Global Change Biology, 11 : 2234-2250.

VIGNAL P., 1956. Les reboisements en Pinus patula de la Haute-Matsiatra. Bois et Forêts des Tropiques, 45 : 15-25.

VITOUSEK P.M., D’ANTONIO C.M., LOOPE L.L., REJMANEK M., WESTBROOKS R., 1997. Introduced species : a significant component of humancaused global change. New Zealand Journal of Ecology, 21 (1) : 1-16.

WALKER L.R., SMITH S.D., 1997. Impacts on invasive plants on community and ecosystems properties. In: Assessment and management of plant invasions. Luken J.O. \& J.W. Thieret (éd.), Springer. Allemagne, p. 69-86. 